\title{
Retraction Note to: The Clinical Implications of Human Telomerase Reverse Transcriptase Expression in Grade and Prognosis of Gliomas: a Systematic Review and Meta-analysis
}

\author{
Jing $\mathrm{Li}^{1} \cdot$ Huiying $\mathrm{Li}^{2} \cdot$ Jihong $\mathrm{Liu}^{3} \cdot \mathrm{Bin} \mathrm{Feng}^{1} \cdot \mathrm{Man} \mathrm{Feng}^{4} \cdot$ Baoyu $\mathrm{Lv}^{5}$. \\ Shaomei Cheng ${ }^{6} \cdot$ Xiangshan Yang $^{4}$
}

Published online: 12 May 2017

(C) Springer Science+Business Media New York 2017

\section{Retraction Note to: Mol Neurobiol (2016) 53:2887-2893 DOI 10.1007/s12035-015-9170-x}

This article has been retracted at the request of the Editor-in-Chief and the Publisher per the Committee on Publication Ethics guidelines. There is strong reason to believe that the peer review process was compromised and the authors have plagiarized parts from the following articles:

The online version of the original article can be found at http://dx. doi.org/10.1007/s12035-015-9170-x

Xiangshan Yang

yangxiangshan163@163.com

1 Dean's Office, Affiliated Hospital of Shandong Academy of Medical Sciences, Jinan, Shandong, China

2 Department of Special Inspection, Affiliated Hospital of Shandong Academy of Medical Sciences, Jinan, Shandong, China

3 The Sixth Department of Intern Medicine, Affiliated Hospital of Shandong Academy of Medical Sciences, Jinan, Shandong, China

4 Department of Pathology, Affiliated Hospital of Shandong Academy of Medical Sciences, Jinan, Shandong, China

5 Department of Nursing, Affiliated Hospital of Shandong Academy of Medical Sciences, Jinan, Shandong, China

6 Department of Gynecology, Affiliated Hospital of Shandong Academy of Medical Sciences, Jinan, Shandong, China
Xiangshan Yang, Shunzeng Lv, Xingtong Zhou, Yuting Liu, Daotang Li, Ranran Shi, Huihui Kang, Jing Zhang, Zhongfa Xu, The Clinical Implications of Transforming Growth Factor Beta in Pathological Grade and Prognosis of Glioma Patients: A Meta-Analysis, Molecular Neurobiology, August 2015, Volume 52, Issue 1, pp 270 276, DOI: 10.1007/s12035-014-8872-9; Received: 6 August 2014

Xiangshan Yang, Shunzeng Lv, Yuting Liu, Daotang Li, Ranran Shi, Zhenyu Tang, Jianzhen Fan, Zhongfa Xu, The Clinical Utility of Matrix Metalloproteinase 9 in Evaluating Pathological Grade and Prognosis of Glioma Patients: A Meta-Analysis, Molecular Neurobiology, August 2015, Volume 52, Issue 1, pp 38-44, DOI: 10.1007/s12035-0148850-2; Received: 20 July 2014

Shunzeng Lv, Bowen Sun, Xiao Zhong, Congxin Dai, Weiping Wang, Xiaochen Ma, Huishu Song, Ranran Shi, Renzhi Wang, The Clinical Implications of Chemokine Receptor CXCR4 in Grade and Prognosis of Glioma Patients: A Meta-Analysis, Molecular Neurobiology, August 2015, Volume 52, Issue 1, pp 555-561, DOI: 10.1007/ s12035-014-8894-3; Received: 20 August 2014

Shunzeng Lv, Congxin Dai, Yuting Liu, Ranran Shi, Zhenyu Tang, Mingzhi Han, Ruixiang Bian, Bowen Sun, Renzhi Wang, The Impact of Survivin on Prognosis and Clinicopathology of Glioma Patients: A Systematic MetaAnalysis, Molecular Neurobiology, June 2015, Volume 51, Issue 3, pp 1462-1467, DOI: 10.1007/s12035-014-8823-5; Received: 26 June 2014 